\title{
Hololens el nuevo perfil de realidad aumentada para la competencia educativa
}

\section{Hololens the new augmented reality profile for educational competition}

\author{
RODRÍGUEZ-CAMPOS, Juan Carlos $\dagger^{*}$, RICO-CHAGOLLÁN, Mariana y GARCÍA-HERRERA, \\ Eduardo
}

Instituto Tecnológico Superior de Irapuato, Carretera Irapuato - Silao Km 12.5 Irapuato, Gto.

ID $1^{\text {er }}$ Autor: Juan Carlos, Rodríguez-Campos / ORC ID: 0000-0002-8079-9654, Researcher ID Thomson: S-7721-2018, CVU CONACYT ID: 263767

ID $1^{\text {er }}$ Coautor: Mariana, Rico-Chagollán / ORC ID: 0000-0001-6942-5902, Researcher ID Thomson: S-7659-2018, CVU CONACYT ID: 691659

ID $2^{\text {do }}$ Coautor: Eduardo, García-Herrera / ORC ID: 0000-0003-2658-6997, Researcher ID Thomson: T-1534-2018, CVU CONACYT ID: 263766

DOI: $10.35429 / \mathrm{JCS} .2019 .7 .3 .13 .19$

Recibido: 12 de Enero, 2019; Aceptado 30 de Marzo, 2019

Resumen

Actualmente en la educación se presentan desafíos que colocan al docente en la necesidad de adquirir nuevos conocimientos para el desarrollo de nuevas habilidades y destrezas didácticas que permitan fomentar actitudes en los alumnos con valoración positiva del pensamiento divergente, creativo y crítico, así como el trabajo multidisciplinario, ordenado y responsable, en la adaptación al cambio. Los Hololens de realidad aumentada es la visión directa o indirecta de un entorno físico del mundo real. Se trata de una tecnología que ayuda a enriquecer la visión de nuestro entorno real completándolo con información del mundo digital, este permite crear imágenes y animaciones ofreciendo una interactividad total con el usuario en tiempo real dentro de un entorno tridimensional. La investigación se enfoca en el uso de una nueva tecnología que permitirá mostrar imágenes tridimensionales e interactuar con grandes volúmenes de datos, los cuales se pueden implementar en el diferente sector incluido la educación, pues debido al avance de esta tecnología y gracias a que los dispositivos móviles se dispone de mayor capacidad de procesamiento y se incluyen cámaras digitales, sensores de última generación y sistemas de localización global, haciendo posible desarrollar sistemas más precisos con realidad aumentada, ya que esta tecnología permite algo que hasta hace poco era impensable, mezclar información digital con escenarios tridimensionales reales, teniendo un impacto en los nuevos perfiles académicos de estudiantes de nivel superior.

Tecnología, Imágenes Tridimensionales, Holo Lens, Realidad Aumentada

\begin{abstract}
Resumen
Currently, in education there are challenges that place the teacher in the need to acquire new knowledge for the development of new skills and didactic skills that can foster attitudes in students with a positive assessment of divergent, creative and critical thinking, as well as work Multidisciplinary, orderly and responsible, in adapting to change. The augmented reality Hololens is the direct or indirect vision of a real-world physical environment. If it is a technology that helps enrich the vision of our real environment complete with information from the digital world, it allows to create images and animations with total interactivity with the user in real time within a three-dimensional environment. The research focuses on the use of a new technology that allows to display three-dimensional images and interact with large volumes of data, which can be implemented in the different sector including education, due to the advancement of this technology and thanks to mobile devices It has greater processing capacity and includes digital cameras, state-of-the-art sensors and global location systems, possible development of more precise systems with augmented reality, since this technology allows something that until recently was unthinkable, mixing digital information with situations real three-dimensional, having an impact on the new academic profiles of higher level students.
\end{abstract}

Technology, Three-dimensional Images, Holo Lens, Augmented Reality

Citación: RODRÍGUEZ-CAMPOS, Juan Carlos, RICO-CHAGOLLÁN, Mariana y GARCÍA-HERRERA, Eduardo. Hololens el nuevo perfil de realidad aumentada para la competencia educativa. Revista de Simulación Computacional. 2019. 3-7: 13-19

\footnotetext{
$\uparrow$ Investigador contribuyendo como primer autor.
} 


\section{Introducción}

Los avances tecnológicos han facilitado el acercamiento a los contenidos educativos, utilizando herramientas creativas y atractivas para los alumnos. De tal manera una de las herramientas tecnológicas son los Holo Lens, que es una percepción de la realidad aumentada y combinándola con las bases de datos crean un entorno con elementos almacenados, para obtener resultados de forma tridimensional. El presente artículo está basado en la Realidad Aumentada, por los alumnos del ITESI, que permite el desarrollando un nuevo conocimiento, tomando como eje central alternativas digitales que por medio de un software brinda la posibilidad de poder observar y crear objetos en tercera dimensión, los cuales pueden ser enfocados o utilizados en diferentes ámbitos laborales tales como la docencia, turismo, procesos industriales, automotrices o mostrar gráficas tridimensionales en tiempo real de la manipulación de la información.

El cambio se ha convertido en un reto universal donde se exige formación y conocimiento especializado en ámbito docente, quienes deben estar preparados para enfrentar las necesidades y demandas de conocimiento para nuevas generaciones de educandos.

Por tal motivo, la educación actual debe adquirir nuevos conocimientos, para el desarrollo de nuevas habilidades y perfiles educativos para la tecnología del futuro. La realidad aumentada es una tecnología, donde la tendencia es representar los resultados de los cálculos en imágenes tridimensionales. Esta nueva tecnología permite crear un nuevo perfil en los alumnos de la carrera de Ingeniería en Sistemas Computacionales del Instituto Tecnológico Superior de Irapuato (ITESI), con el objetivo que el alumno obtenga estructuras mentales previas, que se modifican a través del proceso de adaptación y construye su propia representación de la realidad.

\section{Realidad Aumentada}

Es un paradigma de interacción logrando que el usuario interaccione con el entorno real en el que se halle a través de la emergencia de elementos virtuales. La presentación de un entorno real en el que se combinan elementos reales y virtuales.
La realidad aumentada no sustituye el mundo que nos rodea por uno virtual, sino que agrega objetos virtuales en tres dimensiones a la realidad, incorporando al usuario una nueva información aumentada de la realidad a través de una cámara y una pantalla. No sustituye a la realidad física sino que sobreimprime en ella una serie de datos informáticos. (Ron, Álvarez, \& Núñez, 2013)

Los sistemas de Realidad Aumentada se transforman así en el origen de una serie de tecnologías que pueden convertirse en algo de conocimiento en la sociedad. se trata de dispositivos que, como la realidad virtual, tiene uso inmediato en ingeniería, medicina o biología, pero son susceptibles de aplicarse a cualquier proceso de análisis de la realidad. El termino realidad mixta fue propuesta por Milgram y Kishino en 1994, esta propuesta abarca desde el entorno real a un entorno virtual. (Catala, 2013)

\section{Ventajas de realidad aumentada}

Como ventajas en el uso de realidad aumentada estructuramos que las campañas publicitarias que utilizan la realidad aumentada generan una gran vialidad, y visibilidad de la empresa y del producto. La realidad aumentada es una tecnología utilizada de forma minoritaria lo que provoca un factor sorpresa en los clientes y además favorece la exclusividad de las marcas de lujo también se rompería la barrera tiempo espacio, la educación sería a través de simuladores, fomentando una educación abierta al conocimiento y al software es libre.se beneficiarían varios ámbitos, como la arquitectura, el entretenimiento, el arte, la medicina, las comunidades virtuales y el gobierno.

Nos proporcionaría motivación para proveer de experiencias real con el uso de nuevas tecnologías, permitiendo que el usuario proceda con una experiencia según su propio camino con la facilidad de interacción con el mundo virtual en el que nos encontramos. (López Boch María Acaso, 2012) 


\section{Desventajas de realidad aumentada}

Las desventajas de peligro físico muestra que los teléfonos móviles son actualmente una distracción mientras se conduce un coche, piense en un parabrisas que aumenta direcciones, junto con muchos datos sobre el entorno o imaginar cruzar una calle muy transitada y al mismo tiempo utilizando una interfaz de realidad aumentada para buscar un restaurante y siendo bombardeados con anuncios al mismo tiempo, todo esto, pone en peligro la integridad física de la persona pues lo hace más propenso a tener un accidente. Un spam también se considera como desventaja, porque donde hay una oportunidad de mercado, habrá técnicas de spam, publicidad engañosa y trucos engañar a los consumidores a pagar por cosas que realmente no necesita, o recolectando su información para efectuar grandes estafas después. (Warren Bennett, 2012)

\section{Estereograma}

El estereograma es una ilusión óptica basada en la capacidad que tienen los ojos de captar imágenes desde distintas perspectivas. Estas perspectivas diferentes son captadas de tal forma por el cerebro, que pareciera ser una imagen tridimensional. Los estereogramas se han hecho por años, sobreponiendo dos fotografías tomadas desde ángulos ligeramente distintos, sin embargo en la actualidad han vuelto a la fama gracias a los RDS (Random Dot Stereogram), creados con programas computacionales. Para ver un estereograma, lo principal es entender el resultado esperado. La idea es desenfocar la vista de la imagen, de tal manera que ambas perspectivas sean captadas. Algunos recomiendan mirar al infinito, es decir, fijar la vista en un objeto distante y sin desenfocar, tratar de mirar la imagen. Otros prefieren fijar la vista en un dedo mientras se acerca lentamente a la imagen, $o$ tratar de observar el reflejo de la imagen en un vidrio. Depende de cada persona y de su condición visual. (Héctor, 2013)

\section{Marketing y venta}

Las empresas ven la realidad aumentada como una forma de diferenciarse con respecto a la competencia, ofreciendo al cliente la posibilidad de acceder a experiencias visuales llamativas a través de escenarios virtuales sobre la superficie de ventas, ya sea en probadores y escaparates. Esta tecnología está posicionada para entrar en el sector de consumo de forma generalizada y en los próximos años veremos este tipo de tecnología nos sorprenderá en los escaparates y en los lineales de manera espectacular.
Mediante la realidad aumentada se crea un entorno en que la información y objetos virtuales se fusionan con los objetos reales, ofreciendo una experiencia tal para el usuario que puede llegar a pensar que forma parte de su realidad cotidiana, olvidando incluso la tecnología que le da soporte. Podemos encontrar ejemplos en comercios que han instalado probadores virtuales en sus escaparates aplicaciones que permiten a los transeúntes o internautas probarse prendas de ropa, sin entrar al comercio. (B., 2012)

\section{Medicina}

Lo más probable es que en el futuro las interfaces se produzcan mediante la combinación de realidad virtual y holografía. El aspecto más relevante de la realidad aumentada, que supone una transformación radical de nuestra relación con las imágenes, la realidad y el conocimiento, se refiere a los dispositivos capaces de superponer una imagen sobre la misma realidad. Los sistemas de realidad aumentada se tienen un uso inmediato en ingeniería, medicina, biología, pero son susceptibles de aplicarse a cualquier proceso análisis de la realidad. (Catala, 2013)

\section{Educación}

Las tecnologías de la información y comunicación (TICs) han venido a revolucionar en muchos aspectos la vida del ser humano y el ámbito educativo no ha sido una excepción. El aprendizaje móvil (mobile learning o mlearning) es definido como la impartición de educación y formación por medio de dispositivos móviles.

Se considera una evolución natural del elearning o aprendizaje electrónico, diferenciándose de ese en que el uso de la tecnología móvil confiere flexibilidad al aprendizaje, dado que los estudiantes pueden aprender en cualquier momento y en cualquier lugar. (X. Basogain, 2007)

La Realidad Aumentada (RA) adquiere presencia en el mundo científico a principios de los años 1990 cuando la tecnología basada en los ordenadores de procesamiento rápido las técnicas de renderizado de gráficos en tiempo real y los sistemas de seguimiento de precisión portables, permiten implementar la combinación de imágenes generadas por el ordenador sobre la visión del mundo real que tiene el usuario. 
En el ámbito educativo podemos decir que puede emplearse para contenidos de matemáticas y geometría, biología, física, entre algunas de las materias que se podrían mencionar. Es importante resaltar el hecho de que los estudiantes necesitan imaginar objetos desde diferentes orientaciones, manipular modelos tridimensionales, trasladar mentalmente dibujos de dos a tres dimensiones, en papel o en programas de diseño asistido por ordenador.

Si se les brinda la posibilidad de hacerlo con la Realidad Aumentada también se está desarrollando en ellos habilidades de estructuración del espacio, de resolución de problemas que son necesarias para desarrollar un pensamiento científico y que además posibilitaran el que los contenidos abordados sean más atractivos para ellos. La finalidad de utilizar la Realidad Aumentada en brindar al alumnado entornos de aprendizaje que estimulen la comprensión del espacio que le rodea y más aún si ese es con una visión tridimensional. (Cantero, 2013).

\section{Desarrollo}

El presente trabajo de investigación se enfoca en realizar de aplicaciones de "Hololens" por medio de un software para implementarla en los diferentes campos de aplicación tales como: enseñanza, marketing, venta, viajes, guías turísticas, proceso industrial, automotriz, medicina o red social, etc. De la misma forma, desarrollar un nuevo perfil de conocimiento para el ámbito laboral, por medio de esta nueva tecnología el cual permite realizar imágenes tridimensionales, aumentando el realismo y la comprensión de diferentes resultados matemáticos, modelos o simulaciones dependiendo de su aplicación.

Durante este proceso de investigación se hizo un trabajo colaborativo e integrar los conocimientos de la carrera en Ingeniería en Sistemas Computacionales que realizó la parte de la materia de simulación, matemáticas, sin olvidar los pilares de la programación, todo esto llevado en los laboratorios, aulas y departamentos del Instituto Tecnológico Superior de Irapuato.

El objetivo es realizar, diseñar e implementar imágenes tridimensionales que permita la manipulación de datos por medio de la realidad aumentada y los Hololens.
En base a lo anterior es necesario integrar al alumno para enriquecer su práctica y al docente para enriquecer las formas de enseñanza- aprendizaje y que juntos se dispongan a solucionar problemas que aquejan a la comunidad mediante el uso de investigación y las nuevas tecnologías de la información y comunicación, utilizándolas como herramientas fundamentales. Para esto se realiza la siguiente estrategia didáctica, bajo dos niveles de complejidad.

El primer actividad, consiste en la utilización de software para crear la animación u objeto que debemos representar, donde la imaginación y creatividad del alumno no tiene límite alguno. La siguiente actividad es la programación de marcas, diseñadas para la visualización de la imagen tridimensional, para la representación de resultados, que implica el razonamiento, argumentación para poder implementarlo, todo esto a cargo de los alumnos de la carrera Ingeniería en Sistemas Computacionales.

A continuación en la Tabla 1 se muestran las actividades generadas en cada una de las etapas, y Tabla 2 se muestra los diseños realizados por los alumnos utilizando su creatividad.

\begin{tabular}{|c|c|}
\hline Software & Descripción \\
\hline 3DStudio Max & Diseño de figuras y animación \\
\hline $\begin{array}{ll}\text { Adobe } & \text { Flash } \\
\text { Builder } 4 & \\
\end{array}$ & Compilación y editar el código. \\
\hline Unity & $\begin{array}{l}\text { Unity es un motor de videojuego } \\
\text { multiplataforma creado por Unity } \\
\text { Technologies }\end{array}$ \\
\hline OpenCollada & $\begin{array}{l}\text { Plugin para 3D Studio Max, Maya } \\
\text { o Blender que nos permite } \\
\text { exportar nuestras creaciones. }\end{array}$ \\
\hline $\begin{array}{ll}\text { Adobe } & \text { Flash } \\
\text { Player } & \\
\end{array}$ & Creación de animaciones. \\
\hline $\begin{array}{l}\text { Aumentaty } \\
\text { Author Pack } \\
\text { Setup }\end{array}$ & Procesamiento de escala de grises \\
\hline FLARToolKit & Comprobación de patterns \\
\hline osgART & Calcula de dimensión y rotación \\
\hline
\end{tabular}

Tabla 1 Programas y descripción de actividades 


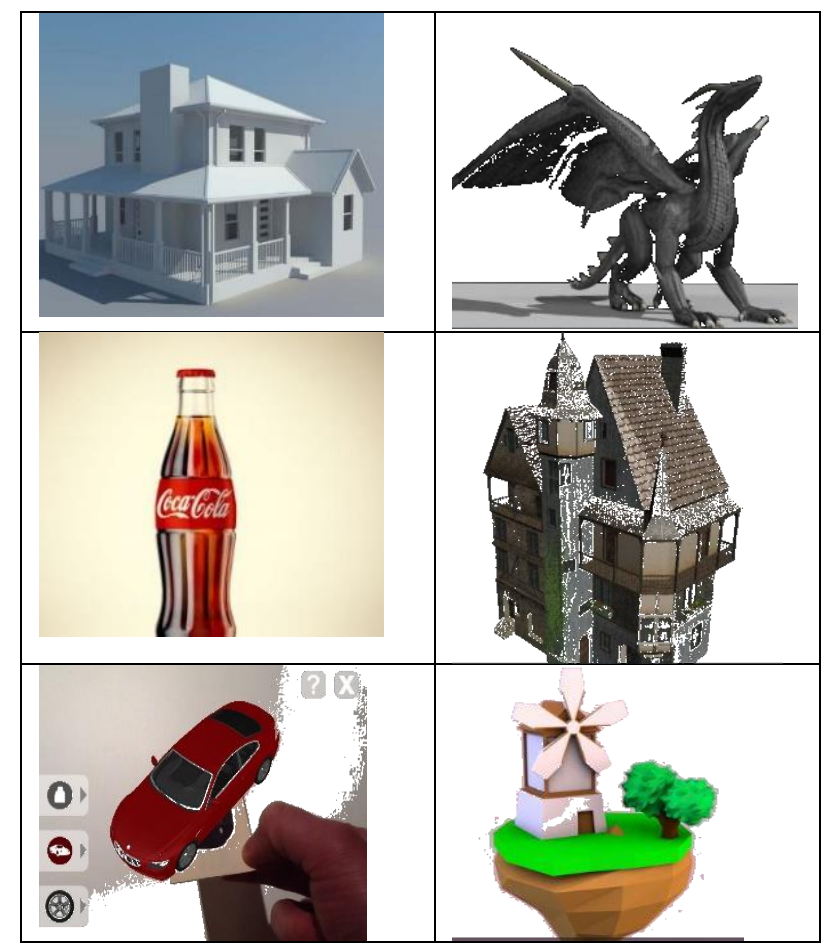

Tabla 2 Diseños realizados

\section{Resultados}

Los resultados se han visto beneficiados porque facilitan la interacción de resultados y diseños tridimensionales. Así mismo el ITESI realiza un seguimiento a egresados con el fin de obtener información del nivel de desempeño de los alumnos en las empresas. Durante el ciclo escolar agosto-diciembre 2017 y enero-junio 2018, después de su egreso (2017), a fin de dar oportunidad a que se involucren en el mercado de trabajo y así, poder obtener información de su desempeño profesional en dichas actividades. Para llevar a cabo el censo, se recurre al muestreo a fin de obtener información confiable, aplicando el muestro aleatorio simple con reemplazo como se muestra a continuación, el cual implica que una vez seleccionada una persona o elemento se regresa al marco, donde tiene la misma probabilidad de ser elegida de nuevo, así la población no altera su tamaño $(\mathrm{N})$. Entrevistando solo a una parte de los egresados, lo que representa la muestra.

$n=\frac{\sigma N p q}{e^{2}(\mathrm{~N}+1)+\sigma p q}$

Donde:

(Tamaño de la población) $\quad \mathrm{N}=285$

(Probabilidad esperada) $\quad \mathrm{P}=0.50$

(Población de fracaso) $\quad \mathrm{q}=0.50$

(Error admisible) $\quad \mathrm{e}=0.05$

(Nivel de confianza) ? $=1.96$

$$
\begin{aligned}
& n=\frac{(1.96)(285)(0.50) 0.50)}{(0.05)^{2}(285+1)+(1.95)(0.50)(0.50)} \\
& n=\frac{(139.65)}{(0.71+0.49)}
\end{aligned}
$$

$n=116$

\begin{tabular}{|l|r|r|r|}
\multicolumn{1}{|c}{ Carrera } & \multicolumn{1}{c}{$\begin{array}{c}\text { Egresados } \\
\text { 2016-2017 } \\
\text { (población) }\end{array}$} & $\begin{array}{c}\text { Egresados } \\
\text { captados } \\
\text { (muestra) }\end{array}$ & $\begin{array}{c}\text { \% } \\
\text { egresados } \\
\text { captados }\end{array}$ \\
\hline Informática & 39 & 16 & $41.02 \%$ \\
\hline Industrial & 59 & 24 & $40.67 \%$ \\
\hline Sistemas & 51 & 21 & $41.17 \%$ \\
\hline Electrónica & 23 & 9 & $39.13 \%$ \\
\hline Bioquímica & 32 & 11 & $34.37 \%$ \\
\hline Electromecánica & 45 & 18 & $40 \%$ \\
\hline Materiales & 16 & 7 & $43.75 \%$ \\
\hline Mecatrónica & 20 & 10 & $50 \%$ \\
\hline
\end{tabular}

\begin{tabular}{|c|c|c|c|c|c|c|}
\hline Carrera & \multicolumn{2}{|c|}{$\begin{array}{l}\text { Completament } \\
\text { e relacionado } \\
\text { (100\% de la } \\
\text { relación) }\end{array}$} & \multicolumn{2}{|c|}{$\begin{array}{c}\text { Parcialment } \\
\text { e } \\
\text { relacionado } \\
\text { (75\% de la } \\
\text { relación) }\end{array}$} & \multicolumn{2}{|c|}{$\begin{array}{c}\text { No } \\
\text { tiene } \\
\text { relación } \\
(50 \% \\
\text { de la } \\
\text { relación } \\
)\end{array}$} \\
\hline Informática & 11 & $73 \%$ & 2 & $13 \%$ & 2 & $13 \%$ \\
\hline Materiales & 4 & $66 \%$ & 1 & $17 \%$ & 1 & $17 \%$ \\
\hline Bioquímica & 9 & $90 \%$ & 1 & $10 \%$ & 0 & \\
\hline Mecatrónica & 7 & $78 \%$ & 1 & $11 \%$ & 1 & $11 \%$ \\
\hline Industrial & 17 & $77 \%$ & 5 & $23 \%$ & 0 & \\
\hline $\begin{array}{l}\text { Electromecáni } \\
\text { ca }\end{array}$ & 16 & $89 \%$ & 2 & $11 \%$ & 0 & \\
\hline Electrónica & 7 & $78 \%$ & 1 & $11 \%$ & 1 & $11 \%$ \\
\hline Sistemas & 16 & $80 \%$ & 4 & $20 \%$ & 0 & \\
\hline
\end{tabular}

Tabla 3 Muestreo de alumnos egresados

Tabla 4 Muestra la relación con respecto al trabajo encontrado

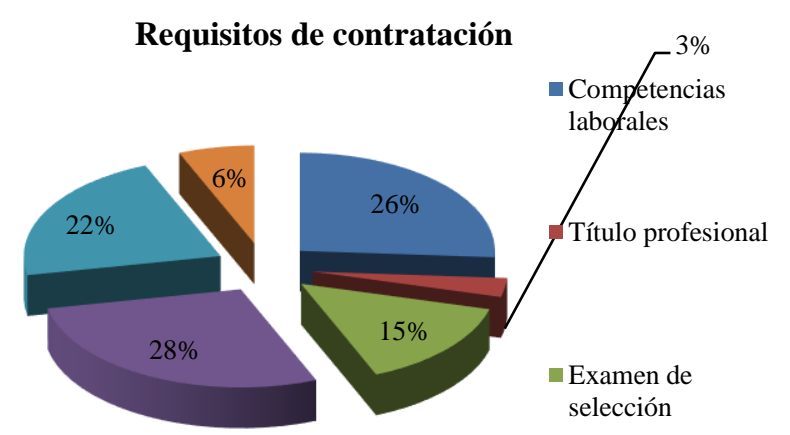

Gráfico 1 Requisitos de contratación 


\section{Evaluación del desempeño profesional por parte del sector productivo}

De acuerdo a la opinión del Sector Productivo, su satisfacción respecto al desempeño de los egresados se considera de la siguiente manera: el $28 \%$ de las empresas establecieron que tiene la satisfacción total con el desempeño de las actividades de los egresados, y un 68\% están muy satisfechos con el desempeño de nuestros egresados, esto nos indica que contamos con un $96.55 \%$ de satisfacción del sector productivo.

\begin{tabular}{|l|r|}
\hline \multicolumn{2}{|l|}{ Desempeño Profesional (Sector Productivo) } \\
\hline a) Excelente (Satisfacción al 100\%) & 28 \\
\hline b) Muy Bueno (Muy satisfecho 90\%) & 69 \\
\hline c) Bueno (Satisfecho 80\%) & 3 \\
\hline d) Regular (Poco Satisfecho 70\%) & 0 \\
\hline e) Pésimo (Insatisfecho 60\%) & 0 \\
\hline No. Egresados & 100 \\
\hline
\end{tabular}

Tabla 5 Nivel de satisfacción del sector productivo

\section{Satisfacción del Sector Productivo}

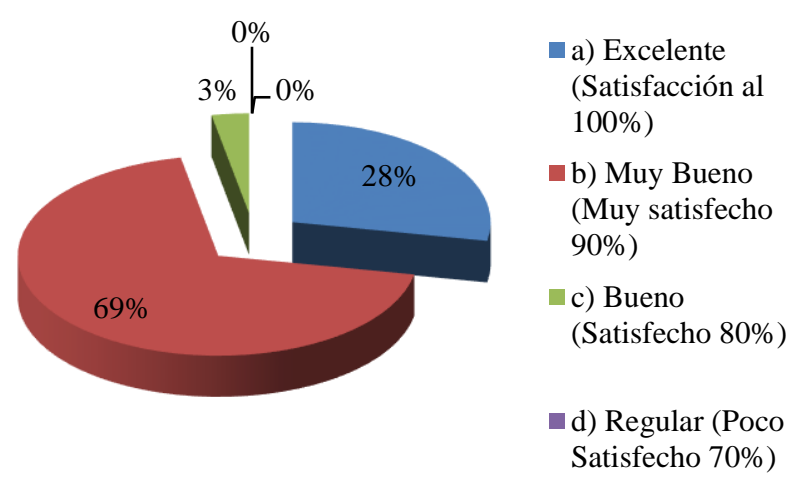

Gráfico 2 Nivel de satisfacción del sector productivo

\section{Conclusiones}

Los Holo Lens nos brinda la oportunidad de ser utilizada en varios ámbitos culturales, marketing, educación, empresa, industria, etc., a lo que ha llegado ser valorada como una atracción en la visualización de información. Esta innovación es utilizada en elementos multimedia que están al alcance, como el diseño gráfico, 3D, video, Audio, imágenes, animaciones, etc., promoviendo sitios de interés en línea. Se puede establecer y vincular a los Holo Lens con las bases de datos, específicamente con los cubos OLAP como una estrategia para obtener resultados con gráficas tridimensionales que se podrían manipular permitiendo de esta manera motivar al alumno para realizar proyectos innovadores que favorezcan su aprendizaje significativo.
Para esto se debe invertir tiempo y esfuerzo ya que el cambio de paradigma exige más tiempo de los que está planeado, sin embargo involucra al mismo tiempo, que el docente esté preparado para enfrentar las nuevas demandas en las técnicas de información, como estudiantil. El docente es parte fundamental para la realización de una metodología exitosa, debido a la incentiva e innovación de procesos para que los alumnos aprendan y puedan desarrollar proyectos por sí mismos, y así crear su propio conocimiento, para desenvolverse en el ámbito laboral y personal. Sin olvidar que el alumno es motivado cuando se tiene un docente con la visión de enseñar, con los pilares de la educación "aprender a aprender, aprender a hacer y aprender a vivir", por lo tanto la práctica docente es uno de los aspectos que se deben tener como prioritarios para el desarrollo de una sociedad.

Por lo tanto es importante el difundir el desarrollo de la realidad aumentada, así como ponerse al día en las nuevas tecnologías, ya que la realidad aumentada cambiará la forma de ver el mundo, dando paso a nuevos perfiles, conocimientos y creatividad, plasmando resultados en tiempo real de forma tridimensional, dando como resultado nuevas tomas de decisión.

\section{Reconocimientos}

Agradezco al ITESI, por brindarme la oportunidad de realizar mis investigaciones. Al Gobierno del Estado de Guanajuato por permitirme los recursos necesarios y realizar la divulgación, comunicación pública este artículo. A mis compañeros de trabajo, sin olvidar a mi jefe de carrera por el apoyo brindado, así como mi amigo, compañero y mentor Dr. Alfredo Lezama Rosas.

\section{Referencias}

B., P. R. (2012). Marketing en el punto de venta, . México: ESIC.

Cantero, J. d. (2013). Entorno de aprendizaje ubicuo con realidad aumentada y tabletas para estimular la comprension del espacio tridimensional. Obtenido de http://www.um.es/ead/red/37/DELATORREet AL.pdf

Catala, J. M. (2013). La imagen compleja. España: Universidad autónoma de Barcelona.

RODRÍGUEZ-CAMPOS, Juan Carlos, RICO-CHAGOLLÁN, Mariana y GARCÍA-HERRERA, Eduardo. Hololens el nuevo perfil de realidad aumentada para la competencia educativa. Revista de Simulación Computacional. 2019 
Héctor, G. M. (2013). Avances en Informática y Sistemas Computacionales. ISBN: 968-574898-5: Universidad Juárez Autónoma de Tabasco, Tomo I.

López Boch María Acaso, J. P. (2012). Las actuales enseñanzas de artes plásticas y diseño. Realidad Aumentada, 25-28.

Ron, R., Álvarez, A., \& Núñez, P. (2013). Los efectos del marketing digital en niños y jóvenes. Madrid: Midac Digital.

Warren Bennett, t. X. (2012). Geo localización y redes sociales. Revista desventajas de la realidad virtual, 257.

X. Basogain, M. O. (2007). Una tecnología emergente. . Realidad Aumentada en la Educación. 